\title{
Las competencias ciudadanas como generadoras de cultura ambiental ${ }^{1}$ \\ Citizen competencies as generators of environmental culture
}

DOI: http://dx.doi.org/10.17981/cultedusoc.9.2.2018.06

Fecha de recepción: 24/06/2018. Fecha de aceptación: 19/09/2018

\author{
Wilman Santander De La Cruz ${ }^{2}$ \\ Viviana Ovalle Lopez; Mary Luz Cervantes Manjarrez; \\ Julio Villamil Ferrer; Asnirelda Rivera Gómez ${ }^{3}$
}

Para citar este artículo

De La Cruz, W., Ovalle, V., Cervantes, M., Villamil, J. y Rivera, A. (2018). Las competencias ciudadanas como generadoras de cultura ambiental. Cultura. Educación y Sociedad 9(2), 67-76. DOI: http://dx.doi.org/10.17981/cultedusoc.9.2.2018.06

\section{Resumen}

Las competencias ciudadanas resultan un componente que promueve hábitos favorables hacia el medio ambiente, razón por la cual el presente estudio se ha centrado en el fortalecimiento de las competencias ciudadanas que permitan la conservación del entorno a través de la promoción de valores, como como la responsabilidad, el respeto y el sentido de pertenencia hacia los recursos ecológicos para el mejoramiento de la calidad educativa, a través de los procesos transversales en las áreas de ciencias, tecnología e innovación. La investigación de tipo cuantitativo, de alcance descriptivo, y se aplicó la encuesta como instrumento de recolección de información. Dentro de los resultados se puede decir que existen una gran motivación por parte de los estudiantes para adquirir competencias ambientales sin embargo se debe seguir desarrollando actividades bajo el direccionamiento de los maestros y que busquen el diseño de estrategias para lograr tal fin.

Palabras clave: Competencias ciudadanas, Educación ambiental; conservación del entorno, participación

\begin{abstract}
Citizen competencies are a component that promotes favorable habits towards the environment, which is why the present study has focused on the strengthening of citizen competencies that allow the conservation of the environment through the promotion of values, such as responsibility, the respect and the sense of belonging to the ecological resources for the improvement of the educational quality, through the transversal processes in the science, technology and innovation areas. The research of quantitative type, of descriptive scope, and the survey was applied as an instrument of information gathering. Within the results it can be said that there is a great motivation on the part of the students to acquire environmental competences, however, activities must continue to be developed under the direction of the teachers and that they should look for the design of strategies to achieve this end.
\end{abstract}

Keywords: Citizenship competencies, environmental education; conservation of the environment, participation

\footnotetext{
${ }^{1}$ Este artículo ha sido derivado del Programa de Fortalecimiento de la Cultura Ciudadana y Democrática CT+I a través de la IEP apoyada en TIC en el Departamento de Magdalena: CICLON.

${ }^{2}$ Líder del grupo de investigación "Ecologistas del Reten”. Correspondencia: Email: profesorwilman@hotmail.com

${ }^{3}$ Docentes pertenecientes al Grupo de investigación “Ecologistas del Reten” de la IED San Juan Bautista. Ciclón Magdalena. Estrategia $\mathrm{N}^{\circ}$ 2. Universidad de la Costa CUC.
}

- The author; licensee Universidad de la Costa - CUC. Cultura, Educación y Sociedad vol. 9 no. 2, pp. 67-76. Julio - Diciembre, 2018 Barranquilla. ISSN 2389-7724 Online 


\section{Introducción}

Una educación de calidad, se centra en la formación de ciudadanos competentes, con un alto grado en valores éticos, respetuoso, que ejerce sus derechos y deberes, amparados en su condición humana. Con alto sentido de responsabilidad social, de tal manera que demuestra una relación de convivencia y respaldo al deber social. En su reto de ser un gran ser humano, ofrece una educación que genere oportunidades, se proyecta lo competitivo y contribuya a cerrar las brechas de inequidad. Una educación centrada en la formación integral propicia una participación en lso contextos educativos, dando libertad a la plenitud de un contexto diverso. (Avendaño, Cortés, Guerrero (2015).

Según (Ortiz, 2009) La Competencias dejan ver la habilidad, cocimientos, pensamientos, carácter que el sujeto deja ver, en sus tres dimensiones cognitivas, expresiva y afectivas. Para que este sujeto pueda ocupar un papel dignamente en la sociedad. Como establece el Ministerio de Educación Nacional en el año 2010, dentro de la Constitución Política del año 1.991 Colombia se comprometió a desarrollar prácticas democráticas para el aprendizaje de los principios de la participación ciudadana en todas las instituciones educativas. Por tanto una competencia refleja una situación subyacente del sujeto, representado en la variedad de hacer validas cosas.

De acuerdo a lo anterior la Institución Educativa Departamental San Juan Bautista, en El Reten Magdalena, se ha fundamentado en fortalecer las Competencias Ciudadanas fuera y dentro del aula de clases. Sin embargo, en la actualidad se evidencian varias problemáticas en los comportamientos de la comunidad educativa frente al manejo inadecuado de las basuras, se ha convertido en un problema tanto de estudiantes y docentes, por ende se ha creado diversas estrategias para dar soluciones a muy corto plazo, persistiendo aun la problemática de las basuras y se han realizado diferentes estudios en contextos educativos similares sobre la importancia de fomentar las competencias ciudadanas, enfocándose en los aspectos relacionados al cuidado del medio y el manejo adecuado de los residuos sólidos en las instituciones educativas.

Razón por la cual surge la necesidad de conocer que impacto ha tenido el proyecto "Por un colegio más limpio" en la IED San Juan Bautista del municipio de el Retén Magdalena. Se observa que algunos de los estudiantes adscritos a este proyecto han mejorado en que ya no depositan las basuras en el suelo, utilizando las canecas que están a su alcance. La dificultad existente tiene relación con un grupo de estudiantes que vienen de otras instituciones educativas y que aún no se han apropiado del proyecto. Se debe persistir en la socialización de esta investigación, para que los estudiantes asimilen el uso adecuado de las canecas según la clasificación de los residuos.

En la medida que los estudiantes asimilen las bondades del proyecto se convertirán en seres humanos comprometidos con el medio ambiente. En un país es fundamental ofrecer formación en ciudadanía a las nuevas generaciones Preguntarse por la formación ciudadana que un país ofrece a las nuevas generaciones. Para el caso de Colombia en donde se están haciendo enormes esfuerzos por "buscar alternativas que nos permitan resolver los conflictos de una manera pacífica, superar la exclusión social, abrir nuevos espacios para la participación 
ciudadana, enfrentar los altos índices de corrupción y lograr relaciones más armoniosas en las instituciones educativas, los lugares de trabajo, los espacios públicos y los hogares de muchos colombianos y colombianas." (Min-Educación, 2002)

Según el documento Estándares Básicos De Competencias Ciudadanas del Ministerio de Educación Nacional de Colombia, aclara que la experiencia de los docentes en el país, debe relacionarse con la transversalidad de varias disciplinas sociales, para abordar la competencia formación ciudadana, cuya competencia busca, que los estudiantes sean cada vez más mejores ciudadanos, capaces de tener comportamientos claros, frente a situaciones culturales, que ameriten transformaciones, por tanto las instituciones educativas, deben llenarse de recursos estratégicos para apuntarle a esta competencia ciudadana. (MEN, s.f)

\section{Degradación Ambiental}

Cuando el abasto disponible de un recurso potencialmente empieza a disminuir. El proceso de deterioro, tanto de la tierra como del aire, del agua, se ven afectados notablemente por el consumo desproporcionado, esta situación afecta nocivamente la salud, la sobrevivencia de las actividades de humanas corren el riesgo de contaminarse. Toda esta situación constituye patrimonio, para los seres humanos trabajar en la inmediatez y se le dé respuesta a necesidades de visibilidad desde el punto de vista para la mejora de la humanidad, la preservación obligatoria de las particulares e influencia del Estado, por preservar el ambiente, y la supervivencia para el desarrollo económico y social en disminuir la degradación social- ambiental (Ambiente).

\section{Derecho.}

El ambiente como variable de influencia para el fortalecimiento de las competencias ciudadanas Según López; Giraldo \& López. (s.f).

Ambiente: Derivado del latín AMBIRE que significa lo que está alrededor de algo. Comprende la delegada piel de nuestro planeta, integrada por los elementos: agua, tierra, aire y fuego solar, es, en fin, el escenario donde interactúan dialécticamente el medio humano y el medio técnico.

Medio Ambiente: Utilizado por primera vez en el año 1833, en Francia, con el significado de medio ambiente exterior. Es el conjunto de factores naturales que permite la vida de los seres humanos y la relación entre ellos. (López, Giraldo, \& López s.f).

Biodiversidad: entendida como la variedad de organismos de cualquier fuente, incluyendo entre ellos los ecosistemas corrientes, marinos y los complejos ecológicos que de él forman parte, la diversidad dentro de cada especie, entre las especies y los ecosistemas.

Impacto Ambiental: Puede ser producida por una determinada acción humana sobre el medio ambiente, por un desastre producido por un hecho de la naturaleza, que afecta a los animales, la salud de las personas, plantas, así como también afecta los bienes del hombre y de la comunidad en general. (López, Giraldo, \& López s.f).

Desarrollo Sostenible: El crecimiento económico la elevada calidad de vida, el bienestar social sin tener en cuenta los recursos naturales renovables, que nos da el sistema, son lo que en última instancia maltratan al medio ambiente. Afectando de alguna manera la satisfacción y progreso del avance razonable del sistema ambiental.

Derecho ambiental: Es la aplicación de la norma, para dar respuesta a las relaciones del hombre con el medio ambiente. (López, Giraldo, \& López s.f).

Fuente: Elaboración propia. (2018). 
La causada por contaminante como el humo que afecta la visibilidad cuando las descargas industriales son abundantes se denominan, contaminación física. Otras sustancias venenosas, fungicidas, plaguicidas que alteran el aire, suelo, agua y al mismo tiempo al hombre, se les llama, contaminación química. Otras que alteran un ecosistema en forma grave, el organismo intruso que a él llega aumentan considerablemente su población, es la contaminación biológica. (Secretaría de Medio Ambiente y recursos naturales. s.f).

El hombre es pieza fundamental a la hora, de mejorar su entorno y preservarlo, puesto que el medio ambiente donde él se encuentra le proporciona los recursos necesarios, para desarrollarse desde el punto de vista intelectual, moral y social. En este sentido la ciencia cobra un impactante avance, para que el hombre camine junto con ella, pues la evolución de las cosas le permite al hombre de hoy romper con paradigmas y travesar los muros que le impiden evolucionar. Para ello debe transformar los recursos que le presenta la ciencia, la técnica y la tecnología, apropiándose de ellos para para presentar innumerables estrategias, para que el pensamiento pueda generar innumerables conocimientos. En este sentido el medio ambiente le presenta al sujeto aspectos naturales y artificiales, que viene hacer la esencia para el bienestar del hombre y para el goce de sus necesidades fundamentales.

Hemos tomado como referente histórico el siguiente escrito, según WERJAYAS, autoridades indígenas de la cultura Uwa, que expresan los siguiente "Si al gobierno les parecen más valiosas sus propias políticas de la naturaleza, en porque en sus políticas puede hacer más cosas que la naturaleza. Que entre mu- chas otras sabe hacer la tierra. Entonces la solución debe ser más fácil para el gobierno; que haga un segundo piso y se la den a las compañías extranjeras para que exploten su petróleo, y nos dejen a nosotros manejar nuestro territorio de acuerdo con las políticas de la naturaleza”. (Sauvé, 1999).

\section{Educación ambiental}

Teniendo como referencia estos conceptos y buscando abordar el mismo desde un enfoque pedagógico, se hace necesario tener en cuenta la definición Novo (2009) expone, en donde define que, "la educación ambiental es un proceso de formación que apunta hacia la toma de conciencia sobre la importancia del medio ambiente, y que busca promover en la comunidad el desarrollo de valores y nuevas actitudes que contribuyan al uso racional y consciente de los recursos naturales y a la solución de los problemas ambientales que enfrentamos a diario".

Es importante tener claridad que a través de este enfoque la educación propone una mirada hacia la transformación de la forma de pensar de una ciudadanía que aún no es consiente del daño que causa en su entorno local o no sabe cómo aminorarlo; esto propone un proceso que se construye paso a paso y que se logra a través de la formación de estas comunidad sobre la importancia del medio ambiente y las consecuencias que sus acciones tienen sobre el medio, para entrar al desarrollo de nuevas actitudes y valores ambientales hoy en día carentes. La anterior definición enmarca el ideal de la educación Ambiental, mirada desde el punto de vista transformador, como lo deben ser bajo cualquier enfoque pedagógico. 
Por tanto los problemas ambientales deben ser tarea para los educadores, cuando estos desde las aulas educativas, admiten que la educación ambiental es una necesidad, que se debe mirar desde la educación, porque la falta de sensibilidad ante los problemas que tiene el medio ambiente, es lo que ha hecho que el individuo cada día más se aleje y se ponga a espalada de ella. Lo que indica que esta problemática como el deteriore del aire, la tala de árboles, todo esto incide notoriamente, en la falta de una educación ambiental, que debe tener como meta el desarrollo de propuestas curriculares que estén articuladas a Proyectos Educativos Institucionales(PEI) y operacionalizarlo de tal manera que se den resultados positivos. (González, 2007).

Según Rengifo, Quitiaquez \& Mora. (s.f) La educación colombiana tiene que darle prioridad a la educación ambiental, que influya en la forma de impartir los conocimientos, actitudes, comportamientos y hábitos frente a la manera de razonar en los programas ambientales. Mediar de tal manera que la concepción y naturaleza del hombre que se tiene de educación ambiental frente a la manera de como armonizar con ella. En este sentido se necesita de educadores comprometidos, con la educación que estén a la vanguardia de apoyar una educación centrada en fortalecer el medio ambiente natural.

Por lo tanto es tarea de la educación, que en los centros educativos se privilegie la educación ambiental y se apoyen en buscar a las instancias necesarias, para que los oriente a cómo interpretar y analizar las reacciones mismas de la naturaleza, entender el entorno natural a utilizarlas, y como aplicar mecanismos de control, donde se puedan emplear normas, para que ellos como individuos se apropian de ella y al tiempo interpretar las leyes que se puedan efectuar y evitar que el hombre la siga destruyendo, de esta manera se le estaría apuntando a una educación que privilegia el medio ambiente, impide su abuso en el consumo y el desuso por no saber cuidarla y protegerla.(2008), en este sentido se debe utilizar el capital humano, para sensibilizarlo frente a los problemas ambientales y llevarlos a que se centren a unos valores culturales del medio ambiente.

Los procesos educativos que incluyen la enseñanza de la educación ambiental pretenden tomar conciencia del individuo y la sociedad de igual forma la capacitación conforman un proceso que permite que los seres humanos y las sociedades desarrollen plenamente la capacidad de conocimiento del mundo y la realidad interpretarlos, explicarlos y vivir sus circunstancias. La educación ambiental promueve el desarrollo y aumenta las posibilidades de la población para emprender su desenvolvimiento. Si bien la educación básica sirve de fundamento para la educación en materia de medio ambiente y desarrollo, ésta última debe incorporarse como parte fundamental del aprendizaje. La educación ambiental es un eje dinamizador para modificar las actitudes de las personas de manera que éstas sean capaces de evaluar los problemas de desarrollo sostenible o sustentable y abordarlos. Según Rengifo, Quitiaquez \& Mora. (s.f).

Cuando se educa frente a las condiciones ambientales se lleva a cabo un proceso de reconocimiento de valores y al tiempo se aclaran los conceptos en la manera de fomentar las actitudes, destrezas, habilidades y aptitudes que permita comprender y apreciar el medio ambiente, frente a los seres humanos, su cultura y la relación con la naturaleza. Es fundamental que cada uno de los que integra las comunidades educativas adquiera conciencia, 
valores, técnicas y comportamientos ecológicos, éticos en consonancia con el desarrollo, haciéndose sensible frente a los elementos que componen la naturaleza y de esta manera se tomen posturas para favorecer la educación ambiental. Naciones Unidas (1992).

La educación ambiental debe tomar en cuenta, los medios naturales y artificiales en su totalidad, es decir debe tener en cuenta lo ecológico, político, tecnológico, social, legislativo, cultural y estético, para que desde este punto de vista, se le de paso a la participación activa de la escuela en la búsqueda de la prevención y solución de los problemas ambientales, analizando las diferencias mundiales, teniendo en cuenta las discrepancias regionales para así dar paso a actividades ambientales actuales y futuras y considerar el desarrollo y crecimiento de la educación para fomentar el valor y la necesidad de la cooperación local, nacional e internacional en la solución de los problemas ambientales locales, que de alguna manera afectan a regiones de poblaciones vulnerables en Colombia, pero que con estrategias internacionales, podrían favorecer la educación ambiental para favorecer las regiones de Colombia.. (Universidad Nacional de Mar de la plata. 2016)

\section{La Formación en Competencias Ciudadanas}

Para Guerrero (2016) es fundamental ofrecer formación en ciudadanía a las nuevas generaciones Preguntarse por la formación ciudadana que un país ofrece a las nuevas generaciones. Para el caso de Colombia en donde se están haciendo enormes esfuerzos por "buscar alternativas que nos permitan resolver los conflictos de una manera pacífica, superar la exclusión social, abrir nuevos espacios para la participación ciudadana, enfrentar los altos índices de corrupción y lograr relaciones más armoniosas en las instituciones educativas, los lugares de trabajo, los espacios públicos y los hogares de muchos colombianos y colombianas." (Min-Educación, 2002)

Según lo fundamental el documento Estándares Básicos De Competencias Ciudadanas del Ministerio de Educación Nacional de Colombia, gracias a las experiencia de muchos docentes en el país, cuando articulan diversas disciplinas y organiza el trabajo, centrado bajo la formación de competencias ciudadana, se dejan principios claros de ciudadanía, lo que indica que los estudiantes se hacen responsables por el logro de una cultura cada vez más clara y agradable, para mejorar estrategias de apoyo a los planes de mejoramiento de cada institución y lograr mejores Proyectos Educativos. (Min-Educación, 2002).

Según (Meza Botero, Escallón Largacha, Rodríguez, Moreno Franco, \& Ucrós Pinzón, 2011) Las competencias ciudadanas establecen una combinación de desempeño, en la que se conjugan habilidades, actitudes y comportamientos del saber ser en contexto. De esta forma se integran las dimensiones del ser para poder conjugarlas. En este sentido se desarrollan competencias ciudadanas para establecer las relaciones y comportamientos con el otro, estableciendo conexiones de análisis, crítico para poder interpretar esquemas y situaciones de la vida social. "De manera evidente, las competencias ciudadanas le apuntan al desarrollo equilibrado y armónico de las habilidades de los educandos, en especial de las capacidades para la toma de decisiones, la adquisición de criterios, el trabajo en equipo, la asunción de responsabilidades, la solución de conflictos y problemas, y 
las habilidades para la comunicación, la negociación y la participación, que deben estar explícitas en todo proyecto educativo institucional."

Para el caso que compete a la investigación se realizara un análisis del impacto del proyecto "por un colegio más limpio" con los estándares que ha configurado el Ministerio de Educación Nacional, los cuales representan dimensiones fundamentales para el desarrollo de las competencias pertinentes.

Frente a lo expuesto las instituciones educativas, dentro de sus planes de estudio. Deben incorporar la competencia ciudadana, como un elemento distintivo del programa, que hará de los estudiantes verdaderos ciudadanos de paz, para enfrentar retos y conflictos que le depara la vida misma. Este tipo de competencia debe desarrollarse de forma transversal para articular situaciones iguales en algunos planes de estudio, Dándole la prioridad a la competencia como formación ciudadana, para que los elementos que la integran, posibilite la diversidad de situaciones que contempla actitudes de comportamientos del ser humano. De esta manera se le da cabida a la identificación de espacios académicos, para que los estudiantes acompañados de sus maestros, defiendan y difundan los derechos de ellos, como elementos esenciales de la convivencia pacífica, para participar de ella, y comprender de forma crítica la diversidad étnica y cultural de un país, como lo plantea la Constitución". (Meza Botero, Escallón Largacha, Rodríguez, Moreno Franco, \& Ucrós Pinzón, 2011)

La convivencia esta llamada al respeto a la vida, a las buenas relaciones con el otro y hacer de los demás derechos humanos una relación justa y equitativa, donde la paz, los principios democráticos y de convivencia, el pluralismo ideológico, la justicia y la equidad, se convierten en el ejercicio para el manejo de la tolerancia y la libertad. Finalmente la formación para la convivencia facilita los comportamientos, en la toma de decisiones, para que de alguna manera abra la puerta ante las cosas de la vida frente y a los problemas que afectan la vida misma, teniendo en cuenta lo económico, político y cultural de una comunidad y/o país.

Para el caso se analizará la participación social entendida como: "La dimensión que tiene por objeto mostrar cómo los valores, prácticas y actitudes culturales tienen una repercusión el sentido de integración, cooperación y emancipación de los individuos y las comunidades, que les conduce a orientar sus acciones. Así, esta dimensión pone de relieve las múltiples formas en que la cultura influye en la preservación y el fortalecimiento de un entorno susceptible de propiciar el progreso social y el desarrollo." (UNESCO, 2014), Pluralidad, identidad y valoración de las diferencias en el estudio y la comprensión critica de la cultura nacional y de la diversidad étnica y cultural de la comunidad educativa, como fundamento de la unidad y de su identidad.

\section{Metodología}

La investigación es de tipo Cuantitativo, ya que permite conocer datos de los participantes en el estudio y proporciona valores numéricos, permitiendo identificar el impacto en el desarrollo de las competencias ciudadanas, con la realización el proyecto "por un colegio más limpio". Según el periodo de tiempo es transversal y se realiza bajo el nivel descriptivo, porque es un proceso que implica observar y detallar el comportamiento de un sujeto sin influir sobre él de ninguna manera, además el estudio es de orden no experimental (Martínez, 2006). 


\section{Población y muestra}

La población participante en este proyecto de investigación, integrado por 200 estudiantes y 11 profesores de la IED San Juan Bautista; es necesaria la aplicación de una entrevista donde se recolecte una información eficaz, se obtengan propuestas que apunten resultados óptimos. Para esta información se tomará una muestra aleatoria de la población demostrada en un $10 \%$ de la misma, en este caso sería 20 estudiantes de la institución.

Técnicas e instrumentos de recolección de la información

Mediante la aplicación de técnicas como la observación y la encuesta, brindó la oportunidad al investigador, tener previo análisis de los problemas hallados, como también las preguntas formuladas, fortalecieron los problemas encontrados. Prepara un guion con preguntas realizado de forma secuenciada y dirigida. Las preguntas son de tipo cerrado y sólo se podrá afirmar, negar o responder una respuesta concreta y exacta sobre lo que se le pregunta. La encuesta tiene 6 preguntas con opciones de respuesta que van de siempre a nunca y 3 preguntas que van de totalmente de acuerdo a totalmente en desacuerdo.

\section{Procedimiento}

Fase (1): Identificación de la problemática a estudiar.

Fase (2): Definición de la población y muestra.

Fase (3): Definición de las variables de estudio.

Fase (4): Fundamentación teórica.

Fase (5): Escogencia de las técnicas e instrumentos de recolección de información.
Fase (6): Aplicación de los instrumentos. Fase (7): Análisis y procesamiento de los datos.

Fase (8): Generación de resultados.

Fase (9): Socialización y discusión de los hallazgos encontrados.

\section{Resultados}

A continuación, se presentan los resultados de la investigación, después de haber aplicado los instrumentos mencionados anteriormente y se desarrollan los resultados obtenidos por cada una de las variables establecidas de acuerdo a las respuestas de los estudiantes participantes, a la descripción del impacto que ha generado la realización del proyecto "por un colegio más limpio en la IEP y la construcción de competencias ciudadanas enfocada a conservación del entorno, de los estudiantes adscritos al programa Ciclón de la IED San Juan Bautista del Municipio de el Retén Magdalena. Permitiendo describir lo siguiente:

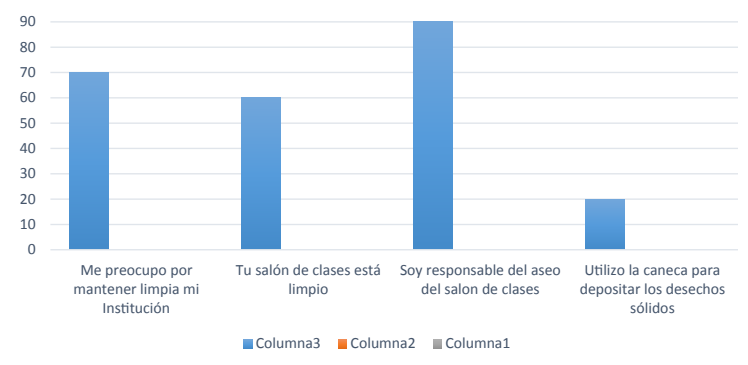

De acuerdo a la gráfica anterior es posible indicar que los resultados permiten ver que frente a la afirmación "Me preocupo por mantener limpia la institución" el 70\% de los participantes efectivamente si se preocupan, seguido de la afirmación "Tu salón de clases está limpio" en donde el 60\% de los estudiantes opinan que sí. Otra de las preguntas fue "Soy responsable del aseo del salón de clases" en donde el $90 \%$ respondió que si, finalmente cuando se les indago sobre 
la afirmación "Utilizo las canecas para depositar los desechos sólidos" el 60\% tuvo una reacción positiva.

Los resultados anteriormente planteados proporcionan una caracterización el estado actual comportamental de los estudiantes participantes, sirviendo como base para dar respuesta a la pregunta problema, e identificando las competencias ciudadanas de los estudiantes en la actualidad, para después de culminado el proceso realizar otra evaluación, en la presente investigación por su parte se obtuvo que los participantes se interesan por el respeto del entorno y de los derechos a vivir en un ambiente sano, a convivir por medio de valores colaborativos y tolerantes, al respeto de las diferencias de los compañeros de clases, a la participación y por consiguiente la decisión en acciones en pro del cuidado de la institución, como elementos predominantes.

(Meza Botero, Escallón Largacha, Rodríguez, Moreno Franco, \& Ucrós Pinzón, 2011) establece que las competencias ciudadanas le apuntan al desarrollo equilibrado y armónico de las habilidades de los escolares, en especial de las capacidades para la toma de decisiones, la adquisición de criterios, el trabajo en equipo, la asunción de responsabilidades, la solución de conflictos y problemas, las habilidades para la comunicación, la negociación y la participación, que deben estar explícitas en todo proyecto educativo institucional"; además son reconocidas como necesarias para generar ambientes apropiados de aprendizaje para un rendimiento académico más óptimo y a la vez lograr el desarrollo de las competencias exigidas por la sociedad actual. (Martìnez, Berthel \& Vergara, 2017).

\section{Conclusiones}

A nivel mundial son conocidos los esfuerzos por establecer compromisos internacionales sobre la conservación del medio ambiente y las políticas necesarias para dicho fin.

Los hallazgos anteriores se encontraron a la luz de del estudio denominado: Impacto del proyecto por un colegio más limpio dentro de la IEP y la construcción de competencias ciudadanas, en el departamento del Magdalena, teniendo como propósito general Describir el impacto que ha generado la realización del proyecto "por un colegio más limpio en la IEP y la construcción de competencias ciudadanas enfocada a conservación del entorno, de los estudiantes adscritos al programa CICLON de la IED San Juan Bautista del Municipio de el Retén Magdalena,

Los estudiantes y docentes reconocen conceptualmente la importancia de la apropiación de las competencias ciudadanas y de poner en práctica las mismas no solo en las instalaciones del colegio sino en la comunidad en general, implementando técnicas innovadoras para tal fin y convirtiéndose en propagadores de la iniciativa.

Los elementos identificados por los estudiantes y adoptados para lograr este propósito tuvieron inicialmente el acompañamiento y orientación de los profesores por lo que se obtuvieron resultados satisfactorios que se continúan presentando dado que su aplicación es permanente.

\section{Referencias}

Avendaño ,I; CortésO Guerrero H. ( 2015) Competencias sociales y tecnologías de la información y la comunicación como factores asociados al desempeño en estudiantes de básica primaria con experiencia de desplazamiento forzado. Diversitas: Perspectivas en Psicología, 11(1). 13-36. 
González. C. (2007). Reciclaje: para la protección del ambiente y los recursos naturales. Recuperado de: http://www. uprm.edu/taubetapi/library/docs/Presentacion $\% 20$ Charla\%20de\%20Reciclaje.pdf

Guerrero. H, y Cepeda, M.(2016) Uso de estrategias pedagógicas para el fortalecimiento de la convivencia escolar de jóvenes vulnerables.http://saber. ucv.ve/ojs/index.php/rev_ped/article/ view/12501.

López, M., Giraldo, N., \& Lopez M. (s.f). Proyecto de Educación ambiental. Recuperado de: http://www.itinelsantuario.edu.co/docs/Proyecto_de_educacion_ambiental_actualizado_1.pdf Martìnez, J. C., Berthel, Y., \& Vergara, M. S. (2017). Síndrome de Burnout en profesores y su relación con el aprendizaje de los estudiantes de básica primaria de una institución educativa oficial de Sincelejo (Colombia). Revista Salud Uninorte, 33(2). 118-128. http:// dx.doi.org/10.14482/sun.33.2.10538

Martínez, M. (2006). La investigación cualitativa (Vol. 9). (R. d. psicología, Ed.) Lima, Perú. Obtenido de http:// revistasinvestigacion.unmsm.edu.pe/ index.php/psico/article/view/4033

Meza, M. C., Escallón, J. E., Rodríguez, C., Moreno, M. F., \& Ucrós, N. (2011). Orientaciones para la institucionalización de las competencias ciudadanas. (M. d. Nacional, Ed.) Bogotá, Colombia: Amado Impresores S.A.S. Obtenido de http://www.colombiaaprende.edu.co/html/micrositios/1752/ articles-314549_recurso_2.pdf

Min-Educación. (2002). Estándares Básicos de competencias ciudadanas. Obtenido de Ministerio de Educación Nacional: https://www.mineducacion. gov.co/1621/articles-116042_archivo_ pdf4.pdf.
Novo, M. (2009). La educación ambiental, una genuina educación para el desarrollo sostenible. Revista de Educación. Universidad Nacional de Educación a Distancia (UNED). Madrid, España. Documento recuperado de: http://www.revistaeducacion. mec.es/re2009/re2009_09.pdf

Ortiz, A. (2009). Desarrollo del pensamiento y las competencias báicas congmitivas y comunicativas. Cuba: Litoral. Obtenido de https://www. autoreseditores.com/book_preview/ pdf/000000728.pdf?1506924227

PNUMA. (1990). Educación ambiental: modulo para la formación de profesores de ciencias y de supervisores para escuelas secundarias. Orelac. Santiago de Chile. Documento recuperado de: http://unesdoc.unesco.org/ images/0007/000714/071480so.pdf

Sauvé, L. (1999). La educación ambiental entre la modernidad y la posmodernidad: En busca de un marco educativo de referencia integrador. Tópicos, 1(2).

Secretaría de Medio Ambiente y recursos naturales. (s.f). Residuos sólidos urbanos: La otra cara de la basura. Documento recuperado de: https:// www.gob. $\mathrm{mx} / \mathrm{cms} /$ uploads/attachment/file/39412/RESIDUOS_SOLIDOS_URBANOS-_ENCARTE.pdf

UNESCO. (2014). Indicadores de cultura para el desarrollo. París, Francia. Obtenido de http://es.unesco. org/creativity/sites/creativity/files/ iucd_manual_metodologico_1.pdf

UNICEF. (s.f). Experiencias urbanas de gestión integral de residuos en 10 municipios de Argentina. Documento recuperado de: https://www. unicef.org/argentina/spanish/EcoclubesbajaWEB.pdf. 